AIAA-2003-1903

\title{
SOME PERFORMANCE TRENDS IN HIERARCHICAL TRUSS STRUCTURES
}

\author{
Thomas W. Murphey* \\ Jason D. Hinkle ${ }^{\dagger}$
}

\begin{abstract}
It has been previously demonstrated that increasing structural complexity can lead to lighter weight structures. However, it is not clear that structural complexity or hierarchy enables lighter weight structures for all architectures and load cases. In this paper, the performance trends in linear truss structures are investigated as a function of self-similar hierarchy order and of loading conditions. The investigations show the order of structural hierarchy resulting in a lightest weight self-similar four longeron solid element truss-column is $2^{\text {nd }}$ (a truss made from trusses) for requirements representative of space structures. The resulting truss-column is typically an order of magnitude lighter than the corresponding $1^{\text {st }}$ order truss-column and two to four times larger in diameter. Long and lightly loaded columns are shown to have the greatest potential for mass reduction with increasing hierarchy. Optimization results for $1^{\text {st }}$ and $2^{\text {nd }}$ order self-similar triangular single-laced double-bay trusses subject to bending strength and stiffness requirements are also presented. A comparison of $1^{\text {st }}$ and $2^{\text {nd }}$ order results show a factor of 30 reduction in truss mass and a simultaneous factor of nine increase in truss diameter.
\end{abstract}

\section{Introduction}

There is evidence to suggest that continually increasing levels of structural hierarchy lead to lighter weight, better performing structures. For example, a tube is stronger than a solid rod of equal weight. Likewise, a truss constructed of tubes is stronger than a truss of solid rods of equal weight. Indeed, topology optimization routines often predict highly latticed solutions and can predict hierarchical structures. ${ }^{1}$ A benchmark topology optimization problem is a horizontal beam subject to a center load. Solutions to this problem show increased latticing as the mesh refinement is increased (Figure 1) and it is standard

\footnotetext{
*Engineering Consultant, AEC-Able Engineering, Inc., Member AIAA

${ }^{\dagger}$ Research Associate, University of Colorado, Member AIAA

Copyright (C) 2003 by Thomas W. Murphey. Published by the American Institute of Aeronautics, Inc. with permission.
}

practice to employ filtering techniques to enforce limits on minimum element sizes.

The Eiffel Tower is a popular example of a hierarchical structure, Figure 2. Hierarchy was employed primarily for manufacturing concerns (only relatively short lengths of steel were available at the time), but the resulting structure achieved an unprecedented level of low effective density. ${ }^{2}$ In the Eiffel Tower, the lowest order building elements $\left(0^{\text {th }}\right.$ order) are rectangular or L-shaped cross-section bars. Columns are built-up from these elements to form trusses with $1^{\text {st }}$ order hierarchy. These trusses are tied together to build the legs of the tower. Each leg has $2^{\text {nd }}$ order hierarchy. The four legs are tied together to form a tower with $3^{\text {rd }}$ order hierarchy.

In deployable beam-like space structures, $1^{\text {st }}$ order hierarchy is most common and is seen in structures built by AEC-Able Engineering (CoilAble, FastMast, AdamMast and stem tube). ${ }^{3}$ Structures with $2^{\text {nd }}$ order hierarchy are also common in the form of trusses built from tubes (AEC-Able SquareRigger solar array, Astro Aerospace AstroMesh antenna, inflatable truss structures by ILC Dover and L'Garde, and the FosterMiller tubular truss ${ }^{4}$ ). In these structures, the $1^{\text {st }}$ order hierarchy is a shell structure and the $2^{\text {nd }}$ is a truss. While they have been discussed, no existing space structures with $2^{\text {nd }}$ order hierarchy and latticing at all levels are known. ${ }^{5}$ Also, spaces structures with hierarchical order greater than $2^{\text {nd }}$ are unknown to the authors.

From a structural performance perspective, one wonders for what conditions structures of increasing hierarchy offer advantages over lower order structures. Similarly, when advantages are perceived, it is important to know how great they are. This paper investigates such issues in an attempt to provide insight into the performance trends in space structures as their hierarchy is increased. The primary parameter under investigation is $n$, the order of structural hierarchy.

The first reference to highly hierarchical space structures appears to be in a short essay by the futurologist, Freeman Dysan, ${ }^{6}$ however, there are few studies that compare the performance of space structures with hierarchy. Mikulas compared the mass efficiency of several column configurations in his seminal paper on the efficiency of long lightly loaded columns, but he was not specifically looking at 
hierarchy. ${ }^{7}$ Interest in structural hierarchy is much more prolific from an effective continuum or material perspective. In reference 2, Lakes provides a review of the work in this area and cites 57 records. Lake looked at the efficiency of various space filling trusses in reference 8. Hierarchical materials are common in nature and have inspired the development of new materials. ${ }^{9,10}$

\section{Preliminary Considerations}

In this paper, an emphasis is placed on self-similar structures. These are structures which are fractal-like in that they obey the same construction rule with each level of hierarchy. Figure 3 illustrates a twodimensional truss construction rule. The $0^{\text {th }}$ order element is a solid rod. The $1^{\text {st }}$ order truss is made from $0^{\text {th }}$ order elements and a $2^{\text {nd }}$ order truss is made from $1^{\text {st }}$ order trusses. This process can be repeated ad infinitum. Self-similar hierarchical structures are not promoted here as offering a structural advantage over more general hierarchical structures. They are considered here for their analytical simplicity and for their clearly defined hierarchical order.

Consider the longeron element of a $1^{\text {st }}$ order truss. This element behaves as a simply supported column subject to both axial stiffness and axial strength requirements. If this column is a slender solid circular rod, it will fail first in an Euler buckling mode. The element could be made stronger in buckling through redistribution of the existing material in a tubular form, without a reduction in axial stiffness. As the tube radius is increased and the wall thickness is decreased (to maintain constant axial stiffness and mass), the strength continues to increase until local wall buckling becomes the first failure mode. The equations for transition between these regions with increasing load were derived with the assumptions shown in Figure 4,

$$
\begin{aligned}
& P<\frac{\pi}{4} \frac{(E A)_{\text {req }}^{2}}{E l^{2}} \quad \rightarrow \quad \operatorname{Rod} \\
& \frac{\pi}{4} \frac{(E A)_{r e q}^{2}}{E l^{2}}<P<\left(\frac{3 \pi}{20}\right)^{1 / 2} \frac{(E A)_{r e q}^{3 / 2}}{l E^{1 / 2}} \rightarrow \text { Tube } \\
& \left(\frac{3 \pi}{20}\right)^{1 / 2} \frac{(E A)_{\text {req }}^{3 / 2}}{l E^{1 / 2}}<P \quad \rightarrow \text { Unclear }
\end{aligned}
$$

These equations are graphed in Figure 5 for a high modulus graphite-epoxy material system and length of $1 \mathrm{~m}$. The longeron design requirement parameters (axial stiffness and compressive strength) are given on the horizontal and vertical axes, respectively. The lines on the graph distinguish between regions where a solid rod is adequate (lowest region), a tube is adequate (middle region), and where higher order structural configurations may be more appropriate (highest region). For the rod and tube regions, lines of constant
$E A$ (vertical) are also lines of constant column mass per length.

Transitions to configurations of increasing hierarchical order occur due to a lack of buckling strength in the longeron. For equal diameter and mass, trusses of increasing hierarchy are only needed when lower order structures are strength limited. As a result, the most appropriate structural form at loads above a local wall buckling limited tube is not clear. Reconfiguration of the material as a $1^{\text {st }}$ order truss would cause a reduction in axial stiffness because material used for battens and diagonals do not contribute to the element axial stiffness. This is quite significant as half the mass of a truss structure is typically in battens and diagonals. Reconfiguration of the material into a cellular cross-section (Figure 6) appears to be more efficient since all material contributes to axial stiffness. However, when unidirectional composite materials are used the difference in axial stiffness contribution between truss and cellular architectures may not be very different -- a tube made from unidirectional composite material requires significant portions of material in the transverse direction to resist local wall buckling.

Actually, truss diameter is not typically fixed and the strength and stiffness of a truss can be significantly increased through an increase in both truss diameter $(l)$ and longeron buckling strength (similarly, an increase in diameter allows a reduction in mass if requirements are only maintained). The increase in truss diameter allows the axial stiffness of elements $(E A)$ to be reduced while keeping truss bending stiffness constant $\left(E I=E A l^{2}\right)$ and herein lies the ambiguity of hierarchy. For equal mass self-similar trusses, increasing hierarchy tends to continually reduce element axial stiffness. At some point, the truss diameter required to compensate for this reduction in element axial stiffness causes an overall increase in truss mass; an optimal hierarchical order is expected for lightest weight self-similar truss structures.

This process of element axial stiffness reduction is dramatic in trusses. Consider a $1^{\text {st }}$ order self-similar truss construction rule that allocates a fraction, $\beta$, of the total truss mass to longerons. For the $2^{\text {nd }}$ order structure, the fraction of mass allocated to axial members is $\beta_{\text {eff }}=\beta^{2}$. In general,

$$
\beta_{\text {eff }}=\beta^{n}
$$

is allocated to axial material. A $3^{\text {rd }}$ order self-similar truss with $\beta=0.5$ allocates only $12.5 \%$ of material to axial stiffness.

The above considerations show that performance trends with increasing hierarchy are not always obvious; regardless of cost and manufacturing 
considerations, an increase in hierarchy is not always better.

\section{A Simple Example}

To illustrate analytically the trends in hierarchical structures, a self-similar hierarchical four longeron truss-column is investigated. This truss is chosen because it is representative of real structures and can be analytically modeled with simple equations. The following characteristics are assumed:

- The truss has four longerons, four member batten frames and four diagonals per bay.

- Each bay is identical (a regular truss).

- Elements are pin jointed.

- Bays are cubic with edge dimension, $l$.

- All lowest order elements have identical solid circular cross sections characterized by a diameter, $d$.

Four bays of a $2^{\text {nd }}$ order example are shown in Figure 7. All $1^{\text {st }}$ order bays in the $2^{\text {nd }}$ order structure are assumed identical and a similar approach is taken for higher orders. For this truss, a parameter that is useful for characterizing the weight per length is the total length of elements per length of bay,

$$
\mu=\frac{4 l+4 \sqrt{2} l+4 l}{l}=13.66
$$

For this truss, the axial material fraction is found as $\beta=4 / \mu=0.29$.

The dimensions of lightest weight hierarchical trusses subjected to axial compressive loads are now derived. The standard procedure for high strength slender structures, and that which is used here, is to equate global and local buckling modes. In doing so, a column length $(L)$ is introduced for the global buckling mode. With the above assumptions, only two design variables are required to specify a $1^{\text {st }}$ order truss ( $l$ and $d)$. This is convenient since there are two design equations (global and local buckling) for sizing the column.

In the following, this sizing procedure is carried out for the hierarchical truss from $0^{\text {th }}$ order (single solid rod) to higher orders and, upon identifying patterns in the resulting performance equations, equations as a function of $n$ are written. A notation in the form of $x_{i j}$ or $x_{i}$ is used. $i$ indicates the top level hierarchical order of the truss and $j$ references an element of $j^{\text {th }}$ order of the truss $(j \leq i)$.

Consider the $0^{\text {th }}$ order solid rod with area $\left(A_{00}\right)$ and cross-section moment-of-inertia $\left(I_{00}\right)$ given by,

$$
A_{00}=\frac{\pi}{4} d_{0}^{2}, \quad I_{00}=\frac{\pi}{64} d_{0}^{4}
$$

where $d_{0}$ is the element diameter. Weight per length of the $\operatorname{rod}\left(w_{00}=\rho A_{00}\right)$ is given by,

$$
w_{00}=\rho \frac{\pi}{4} d_{0}^{2}
$$

where $E$ is Young's modulus and $\rho$ is the bulk material weight density. The Euler column buckling strength $\left(P_{00}\right)$ of this element is,

$$
P_{00}=\frac{\pi^{2} E I_{00}}{L^{2}} .
$$

The lightest weight structure is found by solving Equation (6) for the single design variable, $d_{0}$ with $P_{00}=P$,

$$
d_{0}=\left(\frac{64}{\pi^{3}} \frac{P L^{2}}{E}\right)^{\frac{1}{4}}
$$

The optimized rod weight per length is found through substitution of this design variable solution into Equation (5),

$$
w_{00}=\rho\left(\frac{4}{\pi} \frac{P L^{2}}{E}\right)^{\frac{1}{2}}
$$

A $1^{\text {st }}$ order truss composed of $0^{\text {th }}$ order solid rods is now similarly sized. The truss will have $0^{\text {th }}$ and $1^{\text {st }}$ order element cross-section moment-of-inertias given by,

$$
I_{10}=\frac{\pi}{64} d_{1}^{4}, \quad I_{11}=A_{10} l_{10}^{2}
$$

where $A_{10}$ is the area of a single longeron,

$$
A_{10}=\frac{\pi}{4} d_{1}^{2}
$$

The truss-column weight per length is given by,

$$
w_{11}=\rho \mu A_{10}
$$

and will have global buckling strength $\left(P_{11}\right)$ and local buckling strength $\left(P_{10}\right)$ given by,

$$
P_{11}=\frac{\pi^{2} E I_{11}}{L^{2}}, \quad P_{10}=\frac{\pi^{2} E I_{10}}{l_{10}^{2}}
$$

The lightest weight truss is found by solving Equations (12) for the two design variables, $l_{10}$ and $d_{1}$ with $4 P_{10}=P_{11}=P$,

$$
l_{10}=\left(\frac{1}{\pi^{3}} \frac{P L^{4}}{E}\right)^{\frac{1}{6}}, \quad d_{1}=\left(\frac{8}{\pi^{3}} \frac{P L}{E}\right)^{\frac{1}{3}}
$$

Substitution of these design variables into Equation (11) gives,

$$
w_{11}=\rho \mu\left(\frac{1}{\pi^{3}} \frac{P^{2} L^{2}}{E^{2}}\right)^{\frac{1}{3}}
$$

A similar sizing process was carried out for higher orders of hierarchy. With each order, an additional 
buckling equation and design variable are added so that the system of equations is sufficient to size the structure. This process was carried out sufficiently $\left(6^{\text {th }}\right.$ order) to identify the patterns with increasing $n$. The resulting general equations for element diameter, longest longeron length and structure weight per length are:

$$
\begin{gathered}
d_{n}=\left(2^{3+n-n^{2}} L\left(\frac{P}{\pi^{3} E}\right)^{\frac{n+1}{2}}\right)^{\frac{1}{n+2}} \\
l_{n, n-1}=\left(2^{1-n} L^{n+1}\left(\frac{P}{\pi^{3} E}\right)^{\frac{1}{2}}\right)^{\frac{1}{n+2}} \\
w_{n, n}=\rho \mu^{n}\left(\frac{2^{2\left(1-n^{2}\right)}}{\pi^{2 n+1}} L^{2}\left(\frac{P}{E}\right)^{n+1}\right)^{\frac{1}{n+2}}
\end{gathered}
$$

The column weight per length, which is graphed in Figure 8 , has a minimum with respect to $n$ that typically ranges from two to four. The highest order element length (which is approximately equal to column diameter) and the smallest element diameter equations are graphed in Figures $9 \mathrm{a}$ and $9 \mathrm{~b}$, respectively.

It is of practical interest to determine the relative truss weight reduction with increasing $n$ and the parameters the optimal $n$ is most sensitive to. The ratio of the weight per length of a truss of order $n+1$ to a truss of order $n$ reveals this,

$$
\frac{w_{n+1}}{w_{n}}=\mu\left(2^{-2+\frac{6}{6+5 n+n^{2}}}\right)\left(\frac{P}{\pi^{3} E L^{2}}\right)^{\frac{1}{6+5 n+n^{2}}}
$$

When this ratio first exceeds unity, increasing hierarchy just begins to increase the weight of the structure. This point is the optimal $n$. Equation (18) is a function of $n$ and the single non-dimensional material, geometry and load parameter $P /\left(E L^{2}\right)$ and is graphed in Figure 10 for $\mu=13.66$. The graph shows hierarchy is increasingly beneficial as the non-dimensional parameter decreases. It is only for very long and lightly loaded applications that a hierarchy greater than two results in lighter weight columns. For example, the load supported by a $100 \mathrm{~m}$ (328 ft) column with $E=200 \mathrm{GPa}(29 \mathrm{Msi})$ should be less than $2 \mathrm{~N}(0.45$ lb) before $3^{\text {rd }}$ order hierarchy is employed. The load below which the same column is optimally $2^{\text {nd }}$ order is 2.0E6 N. Considering a short column of $1 \mathrm{~m}$, the load should be less than $200 \mathrm{~N}$ for $2^{\text {nd }}$ order hierarchy to be optimal. These results seem to indicate $2^{\text {nd }}$ order hierarchy is optimal for most reasonable columns.

Where appropriate, an increase in hierarchy from $1^{\text {st }}$ order to $2^{\text {nd }}$ order typically corresponds to a factor of 10 mass reduction and a factor of two to four increase in diameter. The weight reduction and diameter ratio increase as the non-dimensional parameter decreases.

\section{General Optimization}

While they characterize general trends, Equations (15) through (18) may not be representative of efficient space structures. First, space structures are more generally subject to bending stiffness and strength requirements as opposed to column requirements (columns are often considered with space structures because the elements of trusses are columns). Second, structures are not typically limited to the constraints of cubic bays and uniformly equal element diameters. Despite these limitations, the previous example analytically demonstrates general trends with increasing hierarchy and shows the existence of minima in these trends. In the following, more robust numerical optimizations of self-similar trusses are considered for $1^{\text {st }}$ and $2^{\text {nd }}$ order hierarchy. Restrictions on the similarities between elements within a bay are removed and strength and stiffness requirements are more accurately calculated. Straightness imperfection effects are also included. These optimizations are carried out for requirements spanning several orders of magnitude so that the trends are revealed for a large range of space structures.

\section{Triangular Truss Construction Rule}

A three longeron regular truss construction rule is assumed. This truss has been called a triangular double-bay single-laced truss and is shown in Figure 11. The geometry of the truss is characterized by two parameters, bay length $(l)$ and radius $(r)$. The batten and diagonal lengths are related to these parameters by,

$$
\begin{aligned}
& b=r \sqrt{3} \\
& d=\sqrt{l^{2}+b^{2}}=\sqrt{l^{2}+3 r^{2}}
\end{aligned}
$$

The longeron, diagonal and batten stiffness properties are characterized by their effective axial spring stiffnesses: $k_{l}, k_{d}$ and $k_{b}$, respectively. The diagonals of successive bays are assumed to be laced in alternating directions so that the simplest repeating element has length $2 l$. The triangular truss was chosen because it is simple and commonly used in engineering structures. Solid circular $0^{\text {th }}$ order elements are assumed. The details of nodes (joints where longerons, diagonals and battens intersect) are not considered.

\section{Requirements}

The general set of 12 global requirements of a typical beam (stiffness and strength for: axial loads, torsional loads and bending and shear loads about two axes) are reduced to two. First, torsion and axial loads are not considered (-4) because the structure is assumed to act primarily in a bending sense. The beam is also assumed to be isotropic so that bending and shearing are respectively equal for both axes (-4). This leaves 
four parameters (bending and shear stiffness and strength). For a cantilever beam, bending and shear are not independent and this is used to map shear requirements into bending requirements $(-2)$. This reduces the optimized beam requirements to two: bending stiffness and bending strength. For trusses, all lower order elements act as columns and have requirements derived from the global structure requirements.

Bending Stiffness

A cantilever beam is assumed for mapping shear requirements onto bending requirements. The tip displacement $(\delta)$ of a cantilever beam is due to both bending and shearing compliances,

$$
\begin{aligned}
& \delta_{E I}=\frac{P L^{3}}{3 E I}, \quad \delta_{G A}=\frac{P L}{G A} \\
& \delta_{\text {total }}=\delta_{E I}+\delta_{G A}=P L\left(\frac{L^{2}}{3 E I}+\frac{1}{G A}\right)
\end{aligned}
$$

An effective bending stiffness ( $\left.E I_{\text {eff }}\right)$ can be defined that gives $\delta_{\text {total }}$ when used in the equation for $\delta_{E I}$,

$$
\begin{aligned}
\delta_{E I_{\text {eff }}} & =\delta_{\text {total }} \\
\frac{P L^{3}}{3 E I_{\text {eff }}} & =P L\left(\frac{L^{2}}{3 E I}+\frac{1}{G A}\right) \\
E I_{\text {eff }} & =\left(\frac{1}{E I}+\frac{3}{G A L^{2}}\right)^{-1}
\end{aligned}
$$

In the current work, $L$ is assumed to be equal to 10 times the column diameter. This procedure ensures that an optimized beam composed of many bays does not avoid the bending stiffness requirement by deforming through a shear compliance.

\section{Effective Continuum Stiffness Equations}

The analysis of hierarchical structures is potentially daunting because the number of elements increases exponentially with hierarchical order. In the present work, the problem is kept manageable through the assumption of regular trusses. In regular trusses, a relatively simple bay that identically repeats itself can be identified. The behavior of a beam constructed from many bays is then only a function of the behavior of a single bay. Thus, simplified effective continuum equations representing the behavior of a beam of arbitrary length can be written as functions of single bay geometry and element properties.

The direct stiffness method of matrix structural analysis was implemented symbolically in a Mathematica (Wolfram Research Corporation) program to derive such equations. The program generates symbolic equations for the deflections and loads in arbitrary three dimensional pin jointed truss structures. This has been applied to the triangular truss and shown to yield results identical to those obtained by Renton and Noor. ${ }^{11,12}$ The program is readily adaptable to future structural configurations that may become of interest. The effective continuum stiffness equations for the triangular truss are,

$$
\begin{aligned}
E I & =\frac{3}{2} k_{l} l r^{2} \\
E A & =3 k_{l} l \\
G J & =\frac{9 k_{d} k_{l} l r^{4}}{4 l^{2}\left(k_{d}+k_{l}\right)+12 k_{l} r^{2}} \\
G A & =\frac{18 k_{d} k_{l} l r^{2}}{l^{2}\left(k_{d}+4 k_{l}\right)+12 k_{l} r^{2}}
\end{aligned}
$$

Strength Equations

The strength equations are dependent on whether the structure under consideration is the highest order bay (subject to a bending strength requirement) or a lower order element (subject to column strength requirements). Regardless, the structures are subject to both multi-bay and single bay requirements.

Multi-bay strength requirements. A column element must have enough combined bending and shear stiffness to resist global buckling. The equation for global buckling with combined bending and shear compliance is, ${ }^{13}$

$$
P_{c r}=\frac{P_{e}}{1+\frac{P_{e}}{G A}}
$$

where,

$$
P_{e}=\frac{\pi^{2} E I}{L^{2}}
$$

The battens and diagonals must provide enough support to the longerons to enforce local buckling. Also in reference 13, Timoshenko derived an expression for the minimum spring stiffness of equally spaced supports on a long column required to ensure the first buckling mode has a wavelength equal to the support spacing,

$$
\alpha=4 \frac{P_{c r}}{l}
$$

where $P_{c r}$ is the Euler buckling load of the column with length equal to the distance between supports. For the current truss, $\alpha$ is assumed equal to the shear stiffness per length of the face of a bay,

$$
\alpha=\frac{b^{2} k_{b} k_{d} k_{l}}{b^{2}\left(k_{b}+k_{d}\right) k_{l}+k_{b}\left(k_{d}+k_{l}\right) l^{2}}
$$

where $b$ is the length of a batten.

Single bay strength requirements. For pure column loads and bending moments, only the longerons are stressed; diagonals and battens are not loaded and hence, not subjected to strength requirements. The longerons of a column must be stiff enough to resist local buckling and this strength is predicted with 
Equation (23). The load a longeron must support is given by,

$$
F_{l}=\frac{P}{3}
$$

where $P$ is the compressive load applied to the column. For bending loads longerons are stressed according to,

$$
F_{l}=\frac{M}{2 r}
$$

As mentioned, shear strength requirements are mapped into a bending strength requirement. A cantilever beam of length $L$ and root moment $M$ has tip load (maximum shear, $V$ ) given by,

$$
V=\frac{M}{L}
$$

A shear load causes the following maximum element loads,

$$
\begin{aligned}
& F_{l}=\frac{2 V L}{r 3 \sqrt{3}} \\
& F_{d}=\frac{2 V \sqrt{L^{2}+3 r^{2}}}{r 3 \sqrt{3}} \\
& F_{b}=\frac{2 V}{3 \sqrt{3}}
\end{aligned}
$$

Equation (23) is used to ensure each element is stiff enough to support the load given by Equation (30).

A material strength limit of $138 \mathrm{MPa}(20 \mathrm{Ksi})$ is also enforced for all elements. This constraint becomes active only for very low bending stiffness and high bending strength requirements.

\section{Geometric Imperfection Effects}

As element slenderness (length to diameter ratio) increases, straightness imperfections can degrade axial stiffness. An analysis of this stiffness degradation has been previously derived for an initially curved beamcolumn and the solution is applied here. ${ }^{14-19}$ Imperfections are approximated as a sinusoid with half wave-length $l$ and amplitude $a$. It can be shown that the effective spring stiffness of this element is,

$$
k_{o}=\frac{E_{o} A}{l}=\frac{P_{c r}}{2 \varepsilon_{o} l}
$$

where $P_{c r}$ is the Euler buckling load of the element and $\varepsilon_{o}$ is the effective shortening strain due to the imperfection,

$$
\begin{aligned}
& P_{c r}=\pi^{2} \frac{E I}{l^{2}} \\
& \varepsilon_{o}=\frac{1}{1+\frac{4 l^{2}}{\pi^{2} a^{2}}} \approx \frac{\pi^{2}}{4}\left(\frac{a}{l}\right)^{2}
\end{aligned}
$$

The imperfection amplitude to length ratio $(a / l)$ can be attributed to a dimensional stability strain error (potentially due to thermal or manufacturing effects).
A uniform outer fiber strain error $(\varepsilon)$ causes a slender rod to take the shape of a circle. This circle can be approximated as a parabola so that the rod imperfection amplitude to length ratio is given by,

$$
\frac{a}{l} \approx \frac{\varepsilon}{8} \frac{l}{r}=c \frac{l}{r}
$$

In the current work, the strain error is assumed to be $0.012 \%$ so that $c=0.000015$. This corresponds to a $120{ }^{\circ} \mathrm{C}$ temperature change in a unidirectional graphite material with coefficient of thermal expansion of $1 \mu \varepsilon /{ }^{\circ} \mathrm{C}$. This imperfection is assumed for all elements and all hierarchical levels.

The total effective spring stiffness of an element is that due to a combination of stretching and bending,

$$
k_{\text {eff }}=\left(\frac{1}{k}+\frac{1}{k_{o}}\right)^{-1}
$$

For a solid rod element, $k$ is given by,

$$
k=\frac{E A}{l}
$$

A reduction in local buckling strength occurs in imperfect columns due to non-uniform longeron loading. This strength reduction mechanism is not considered here.

Design Variables

The optimization method considers a beam of arbitrary length subject to both bending stiffness and bending strength requirements and finds the lightest weight per length bay that satisfies the requirements. For the $1^{\text {st }}$ order system, bay length and diameter are optimized along with longeron, batten and diagonal diameters (five parameters total). The effective beams are assumed regular in that member diameters do not vary along their length. For the $2^{\text {nd }}$ order system, bay length and diameter are optimized for the $1^{\text {st }}$ and $2^{\text {nd }}$ order elements (eight parameters) along with (independently) the diameters of the three $1^{\text {st }}$ order elements of the three $2^{\text {nd }}$ order elements (nine parameters) for a total of 17 optimization parameters. This optimization procedure was carried out for a range of bending stiffness and strength requirements.

\section{Results}

The $1^{\text {st }}$ and $2^{\text {nd }}$ order triangular truss optimization results for weight per length, bay radius, $0^{\text {th }}$ order longeron diameter and $0^{\text {th }}$ order longeron slenderness are shown in Figures 12 and 13, respectively.

$11^{\text {st }}$ Order Optimization Results

Below an $E I$ to $M$ ratio of $100 \mathrm{~m}$, truss weight per length and longeron diameter are independent of $E I$. In this region strength requirements drive the truss design. Above an EI to $M$ ratio of $10^{6} \mathrm{~m}$ the 
truss is more stiffness driven and the strength requirements are not active.

Longeron slenderness exhibits two regions of relatively constant values of 30 and 115 . The transition between the two regions is relatively narrow but corresponds to the cases where both stiffness and strength constraints are active. This interesting result implies structures should be either slender $(l / d=115)$ or stout $(l / d=30)$, determined by the ratio of $E I$ to $M$.

$2^{\text {nd }}$ Order Optimization Results

The $2^{\text {nd }}$ order truss optimization results exhibit similar regions of independence of $E I$ and $M$ requirements, but the transition region is much broader. Both $E I$ and $M$ constraints are active over most of the plots. Interesting minima are observed on the longeron diameter contours lines.

$1^{\text {st }}$ to $2^{\text {nd }}$ Order Truss Trends

The ratio of $2^{\text {nd }}$ order truss weight per length to $1^{\text {st }}$ order truss weight per length is shown in Figure 14. The optimized $2^{\text {nd }}$ order structure weight per length ranges from 0.010 to 0.035 times that of the equivalent optimized first order structure. The greatest weight savings is for $1^{\text {st }}$ order structures that are farthest from a point where both stiffness and strength constraints are active. Thus, structures with high EI and low $M$ requirements or low $E I$ and high $M$ requirements receive the greatest weight reduction with an increase to $2^{\text {nd }}$ order hierarchy.

The ratio of $2^{\text {nd }}$ order truss diameter to $1^{\text {st }}$ order truss diameter is graphed in Figure 15. While this ratio ranges from 1 to 14 , there is a broad plateau at 9. Thus, an optimized $2^{\text {nd }}$ order truss is typically an order of magnitude larger than an optimized $1^{\text {st }}$ order truss.

\section{Conclusions}

Space structures often have stringent size requirements and are lightly loaded. Such structures are more stiffness limited than strength limited, precluding the need to incur the complexities of increased hierarchy. ${ }^{20}$ However, when an increase in diameter is allowed, hierarchy greater than $1^{\text {st }}$ improves performance. In the assumed solid element trusscolumn, the optimal hierarchy is typically $2^{\text {nd }}$. A long, lightly loaded truss-column has the greatest potential to benefit from an increase in hierarchy. The weight efficiency of the hierarchical truss was shown to be a function of the non-dimensional parameter $P /\left(E l^{2}\right)$. The transition from $1^{\text {st }}$ to $2^{\text {nd }}$ order hierarchy occurs for for values of this parameter near $10^{-9}$. The transition from $2^{\text {nd }}$ to $3^{\text {rd }}$ order hierarchy occurs for values of this parameter near $10^{-15}$. Very large solar sails are potentially in the load range where $3^{\text {rd }}$ order hierarchy results in the lightest weight structure.
General optimization of $1^{\text {st }}$ and $2^{\text {nd }}$ order solid element trusses subject to bending requirements indicate optimized $2^{\text {nd }}$ order trusses are two orders of magnitude lighter than $1^{\text {st }}$ order trusses. They are also typically nine times larger in diameter. These results differ significantly from those for the column optimizations. It is hypothesized that the differences are due to the change in requirements (bending vs. compressive strength) and a higher effective axial mass fraction $(\beta)$ for the more generally optimized beams.

Several assumptions were made in these studies that have the potential to influence the results. For example, solid element as opposed to tubular element structures were assumed. The structures were assumed self-similar trusses as opposed to allowing lattice and shell architectures in the same structure. Relatively non-conservative imperfection amplitudes were assumed. Also, a high modulus, low density and high strength material system was assumed. These are all interesting aspects of the current study and warrant investigation. It is hoped that such can be accomplished in future work.

\section{References}

1 Bendoe, M.P. and Sigmund, O., Topology Optimization: Theory, Methods and Applications, Springer-Verlag, Berlin, 2003.

2 Lakes, R., "Materials with Structural Hierarchy," Nature, Vol 361, pp. 511-515, February 1993.

3 AEC-Able Engineering Inc., http://www.aecable.com.

4 Hinkle, J.D., Peterson, L.D., and Warren, P.A., "Structural Performance of an Elastically Stowable Tubular Truss Column," Proceedings of the 43rd Structures, Structural Dynamics and Materials Conference, AIAA-2002-1555, Denver, Colorado, April, 2002.

5 Mikulas, Martin M., Bush, Harold G., and Card, Michael F., "Structural Stiffness, Strength and Dynamics Characteristics of Large Tetrahedral Space Truss Structures," NASA TMX-74001, March, 1977.

6 Dyson, F.J. in Perspectives in Modern Physics: Essays in Honor of Hans Bethe, Marshak. R.E. (ed.) pp. 641-655, Wiley Interscience, New York, 1966.

7 Mikulas, M. M., "Structural Efficiency of Long Lightly Loaded Truss and Isogrid Columns for Space Applications,” NASA TM-78687, July 1978.

8 Lake, M. S. and Klang, E.C., "Generation and Comparison of Globally Isotropic Space-Filling Truss Structures," AIAA Journal, Vol. 30, No. 5, May 1992. 
9 Anonymous, Hierarchical Structures in Biology as a Guide for New Materials Technology, National Academy Press, Washington, D.C., 1994.

10 Gibson, L.J. and Ashby, M.F., Cellular Solids, Pergamon, Oxford, 1988.

11 Renton, J. D., Elastic Beams and Frames, Camford Books, books@camford.org.uk, 2000.

12 Noor, A. K. and Mikulas, M. M., "Continuum Modeling of Large Lattice Structures - Status and Projections," NASA TP-2767, 1988.

13 Timoshenko, S. P. and Gere, J.M., Theory of Elastic Stability, $2^{\text {nd }}$ edition, McGraw-Hill Publishing Company, 1961, pp. 28, pp. 32.

14 Crawford, R.F. and Hedgepeth, J.M., "Effects of Initial Waviness on the Strength and Design of Built-up Structures," AIAA Journal, Vol. 13, No. 5, May 1975.

15 Anderson, M.S., "Nonlinear and Tangent Stiffness of Imperfect Beam Columns," NASA TM 84497, Dec. 1982.
16 Fichter, W. B. and Pinson, M. W., "LoadShortening Behavior of an Initially Curved Eccentrically Loaded Column," NASA TM-101643, 1989.

17 Lake, M. S. and Georgiadis, N., "Analysis and Testing of Axial Compression in Imperfect Slender Truss Struts," NASA TM 4174, 1990.

18 Murphey, T. W. and Mikulas, M. M., "Nonlinear Effects of Material Wrinkles on the Stiffness of Thin Polymer Films," Proceedings of the the $40^{\text {th }}$ Structures, Structural Dynamics and Materials Conference, St. Louis, Missouri, April 1999.

19 Hinkle, Jason D., Warren, Peter A. and Peterson, Lee D., "Structural Performance of a Gossamer Isogrid Column with Initial Geometric Imperfections" AIAA-2001-1682. Proceedings of the $42^{\text {nd }}$ Structures, Structural Dynamics and Materials Conference, Seattle, Washington, April, 2001.

20 Hedgepeth, J. M., "Critical Requirements for the Design of Large Space Structures," NASA CR 3484, 1981.

a)

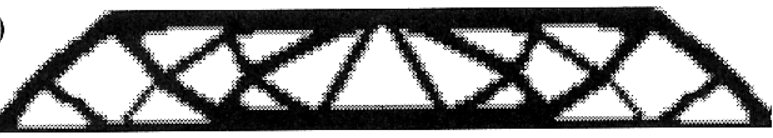

b)
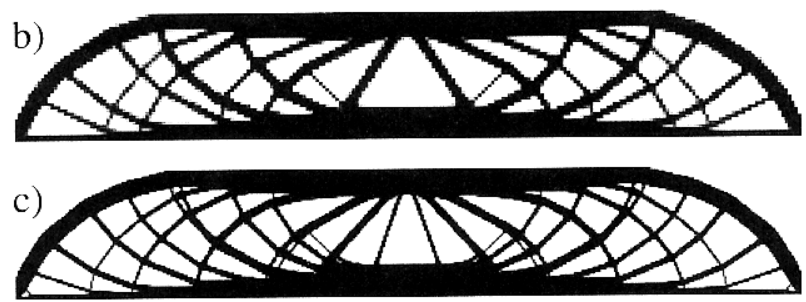

Figure 1: Increased latticing in topology optimization solutions for a beam with center load as mesh density is increased (reference 1). 


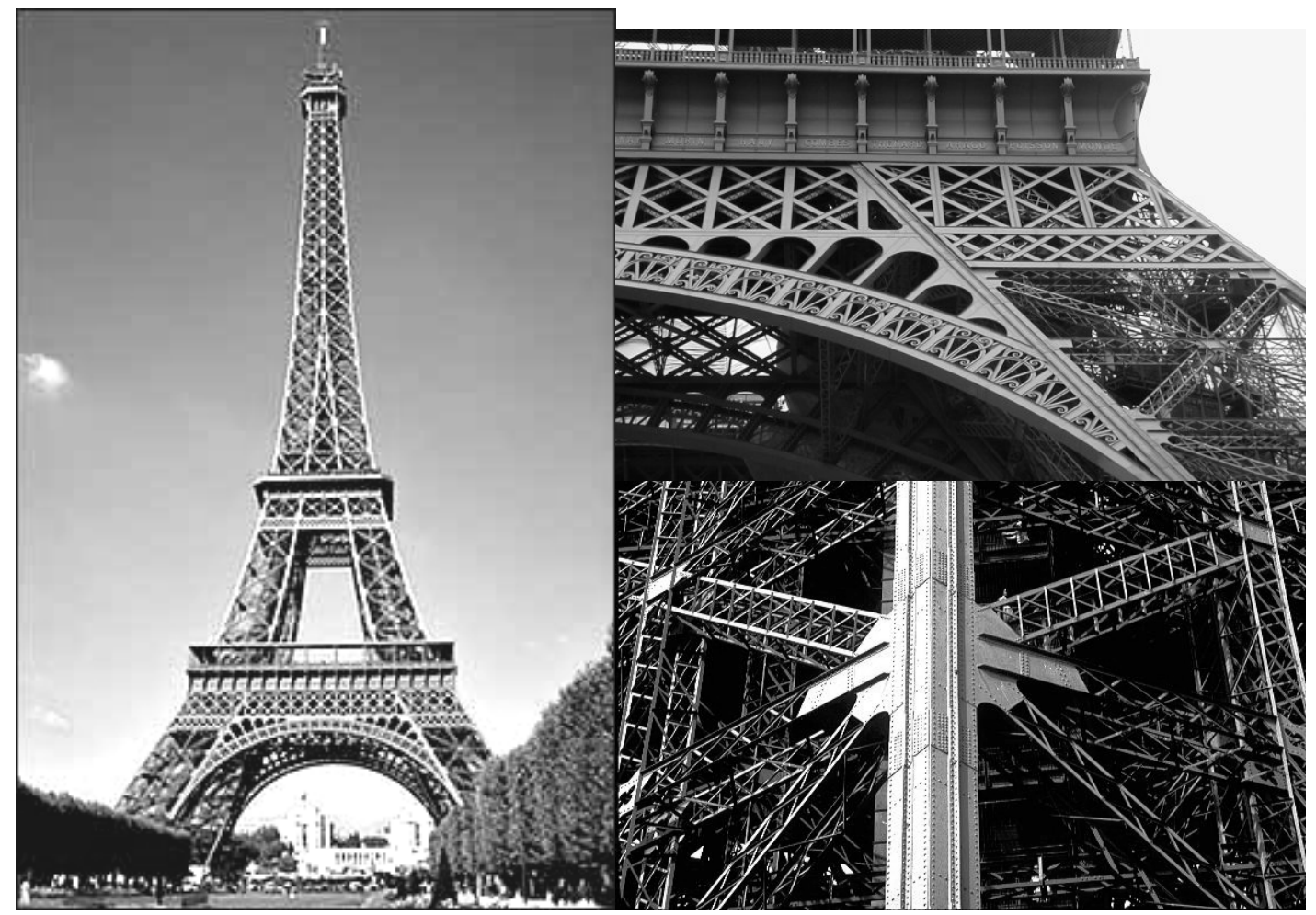

Figure 2: Photographs showing the 3 levels of hierarchy in the Eiffel Tower (Paris, France).
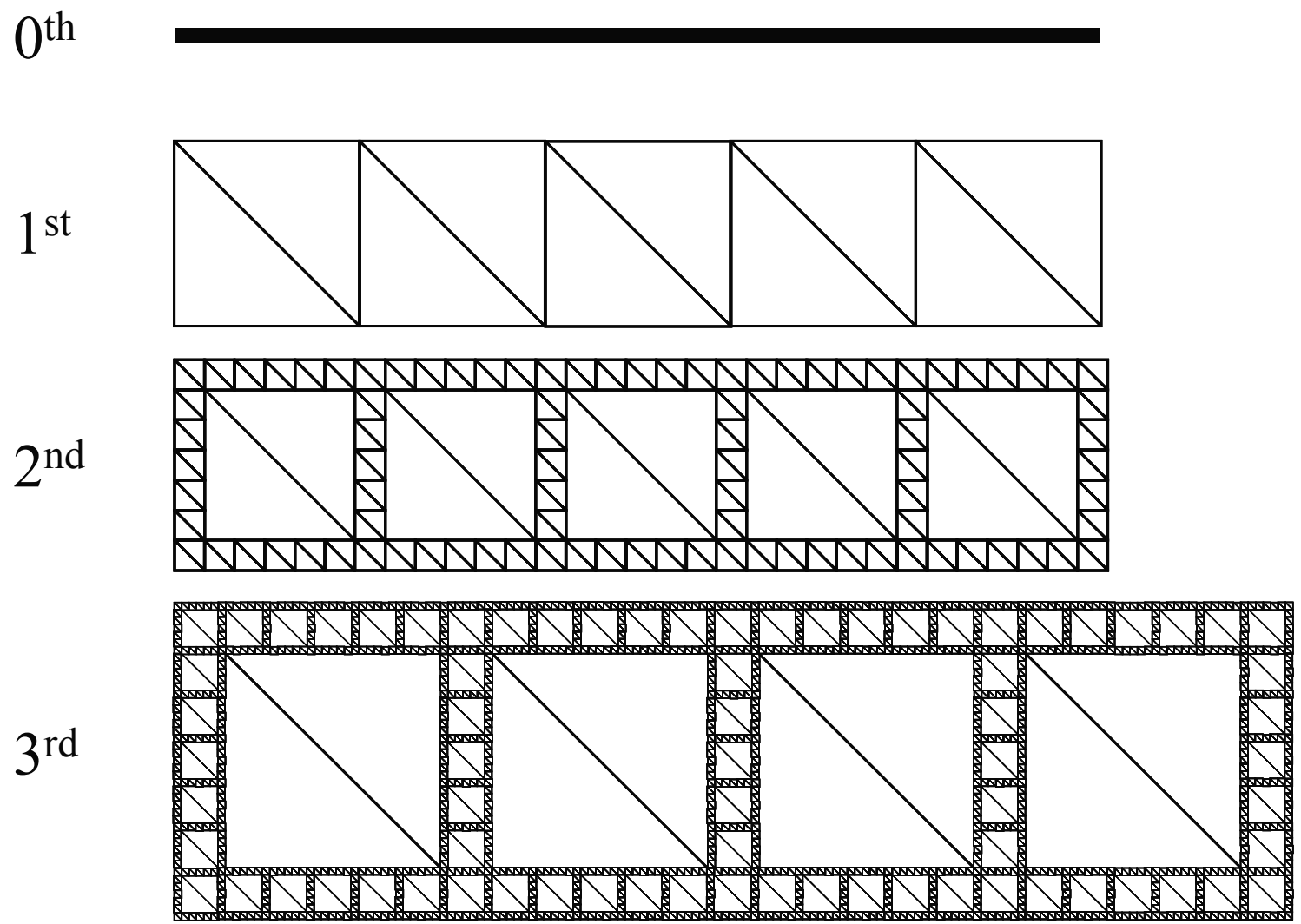

Figure 3: Increasing structural hierarchy with a two-dimensional self-similar construction rule. 


\section{Rod to tube transition equation derivation:}

Cross-section area and inertia: $A=\frac{\pi}{4} d^{2}$ and $I=\frac{\pi}{64} d^{4}$

Axial stiffness requirement determines required $d: E A=(E A)_{\text {req }} \rightarrow d^{2}=\frac{4}{\pi} \frac{(E A)_{\text {req }}}{E}$

Transition load determined by Euler buckling constraint: $P_{\text {trans }}=\frac{\pi^{2} E I}{l^{2}}=\frac{\pi}{4} \frac{(E A)_{r e q}^{2}}{E l^{2}}$

Tube to higher order transition equation derivation:

Cross-section area and inertia: $A=\pi d t$ and $I=\frac{\pi}{8} d^{3} t$

Axial stiffness and global buckling requirements determine tube thickness and diameter:

$$
\left\{\begin{array}{c}
P_{\text {trans }}=\frac{\pi^{2} E I}{l^{2}}=\frac{\pi^{3} E d^{3} t}{8 l^{2}} \\
(E A)_{\text {req }}=E \pi d t
\end{array}\right\} \rightarrow\left\{\begin{array}{c}
d=\frac{2 l}{\pi} \sqrt{\frac{2 P_{\text {trans }}}{(E A)_{\text {req }}}} \\
t=\frac{1}{2 E l} \sqrt{\frac{(E A)_{\text {req }}^{3}}{2 P_{\text {trans }}}}
\end{array}\right\}
$$

Transition load determined by local buckling constraint:

$$
P_{\text {trans }}=A \frac{12}{10} \frac{E t}{d} \rightarrow P_{\text {trans }}=\left(\frac{3 \pi}{20}\right)^{1 / 2} \frac{(E A)_{\text {req }}^{3 / 2}}{l E^{1 / 2}}
$$

Figure 4: Rod-tube-higher order column transition equation derivations.

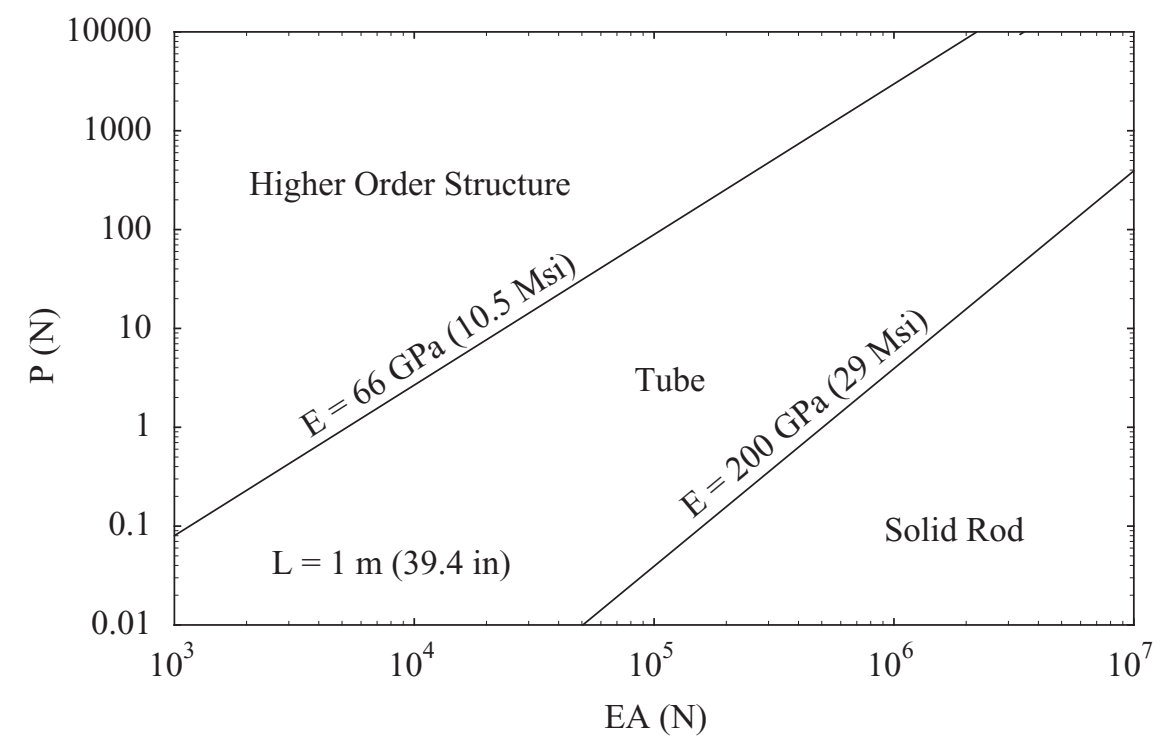

Figure 5: Simplest longeron form as a function of stiffness and strength requirements.

Figure 6: A $2^{\text {nd }}$ order cellular beam cross-section.

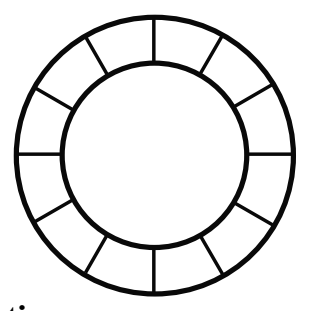




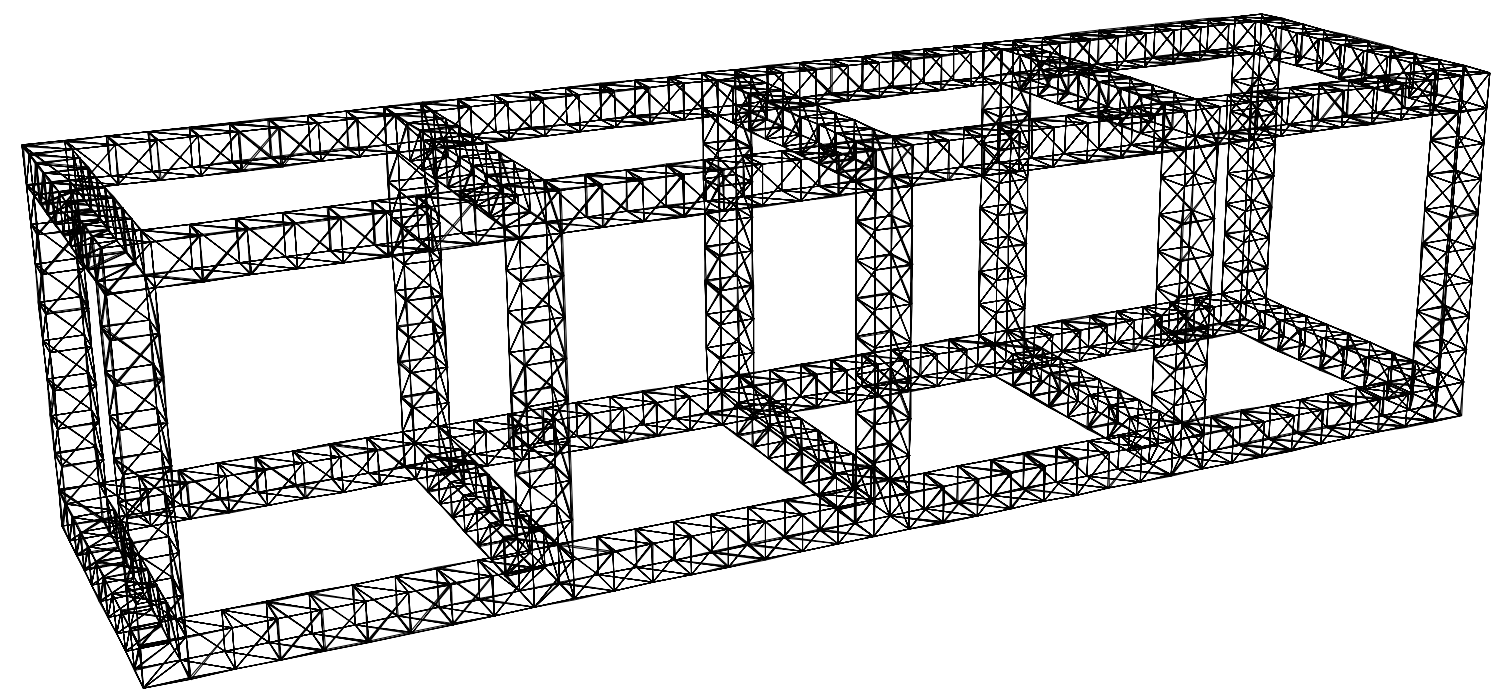

Figure 7: $2^{\text {nd }}$ order truss-column $\left(2^{\text {nd }}\right.$ order diagonals not shown).

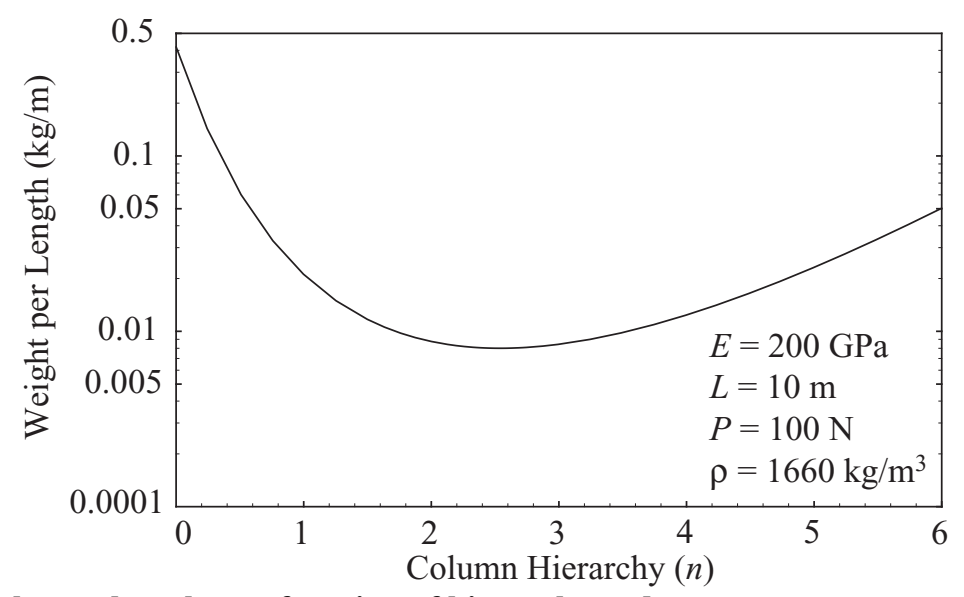

Figure 8: Column weight per length as a function of hierarchy order.

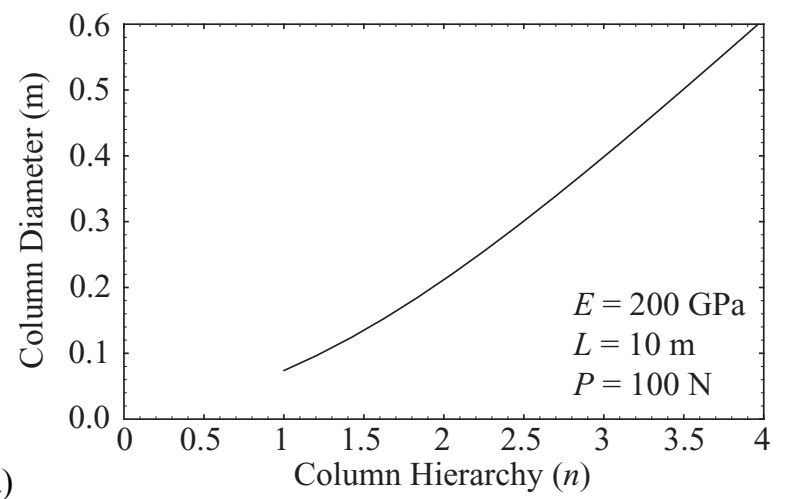

.)

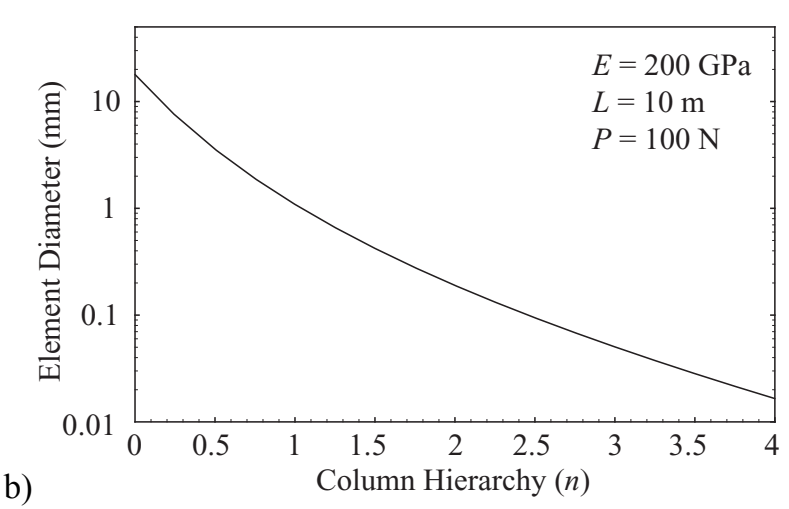

Figure 9: Column diameter (a) and smallest element diameter (b) as a function of hierarchy order. 


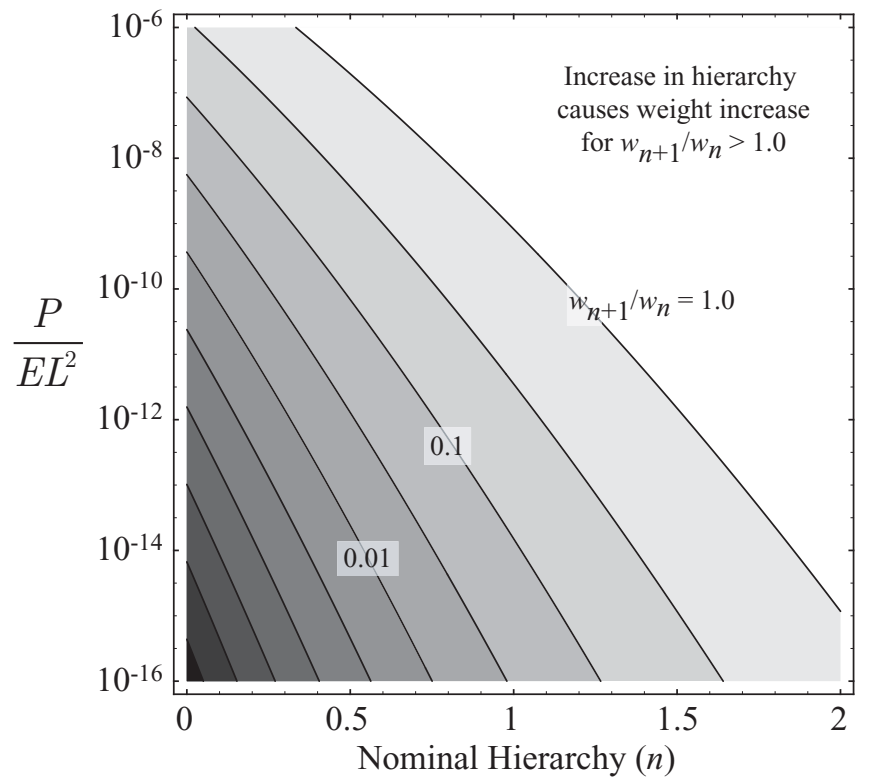

Figure 10: Ratio of $n+1$ order structure weight per length to $n$ order structure weight per length.

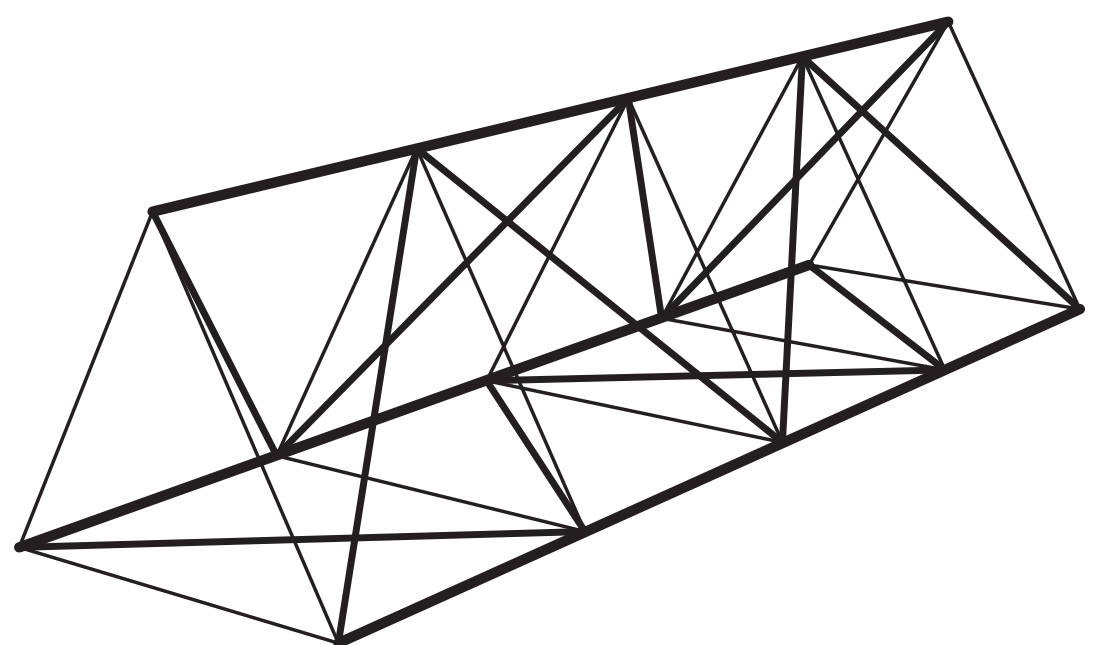

Figure 11: Four bays of the triangular double-bay single-lacing truss. 

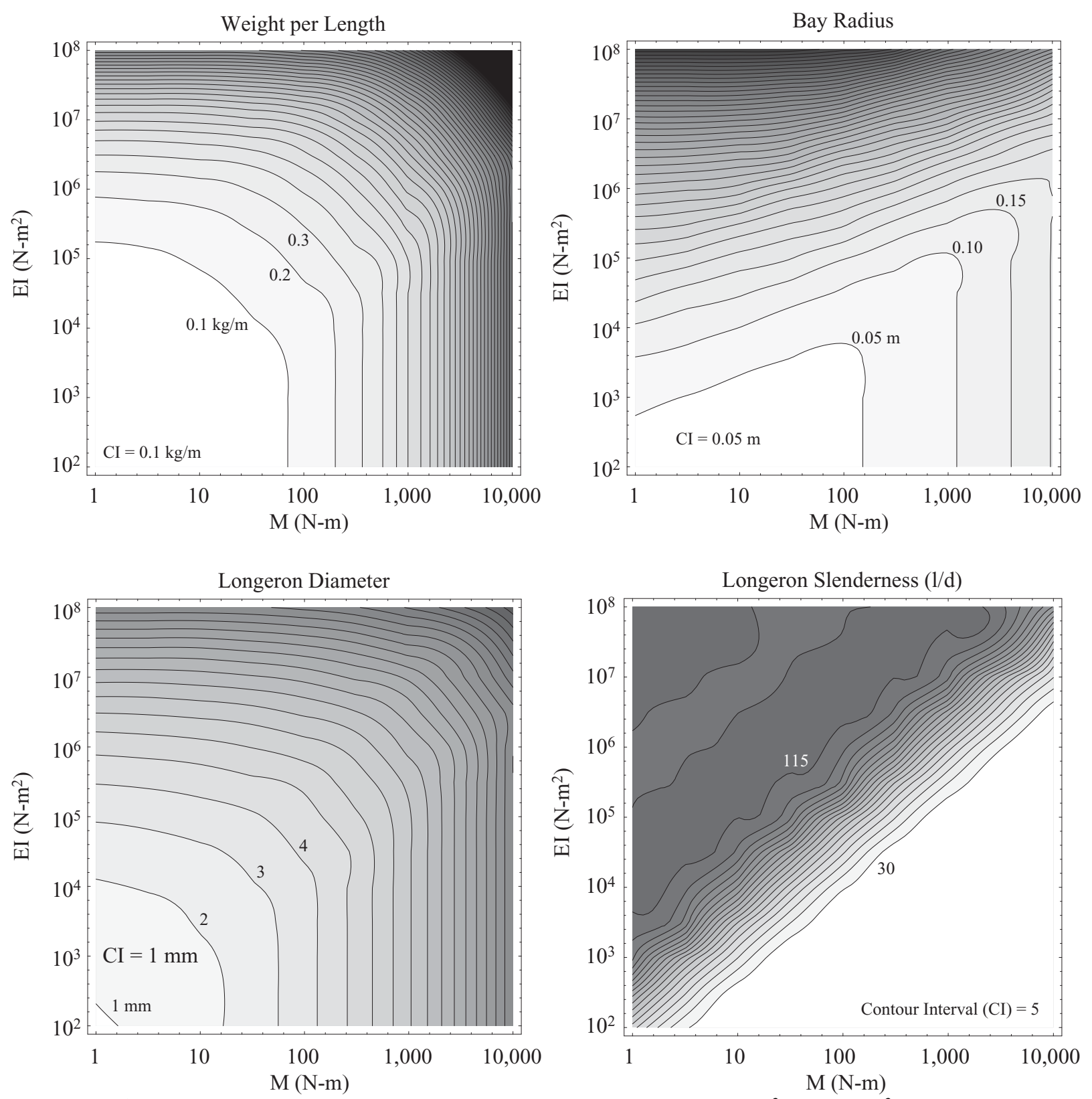

Figure 12: $1^{\text {st }}$ order optimization results $\left(\mathrm{E}=200 \mathrm{GPa}(29 \mathrm{Msi}), \rho=1660 \mathrm{~kg} / \mathrm{m}^{3}\left(0.06 \mathrm{lb} / \mathrm{in}^{3}\right)\right)$. 

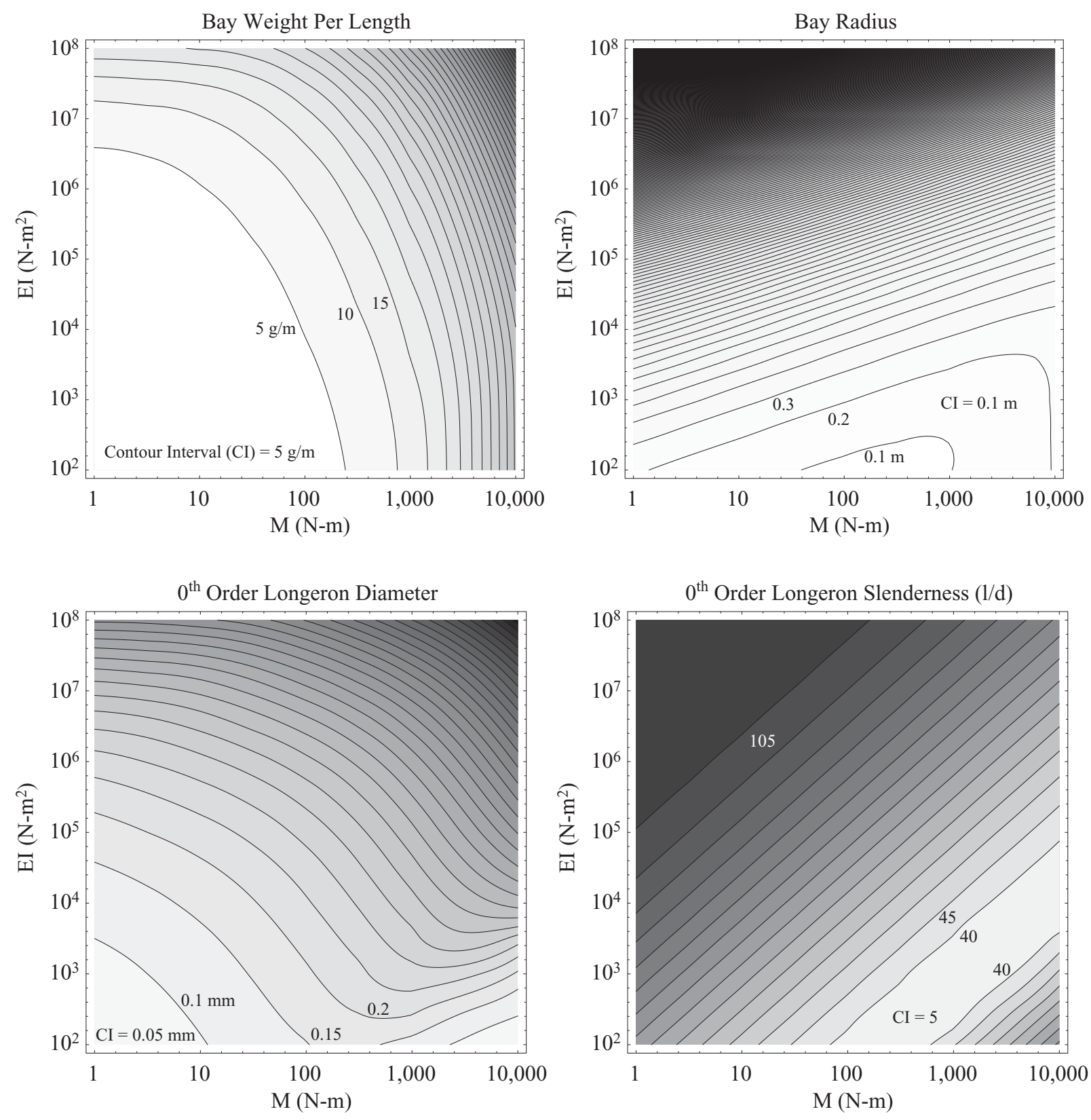

Figure 13: $2^{\text {nd }}$ order optimization results $\left(E=200 \mathrm{GPa}(29 \mathrm{Msi}), \rho=1660 \mathrm{~kg} / \mathrm{m}^{3}\left(0.06 \mathrm{lb} / \mathrm{in}^{3}\right)\right)$. 


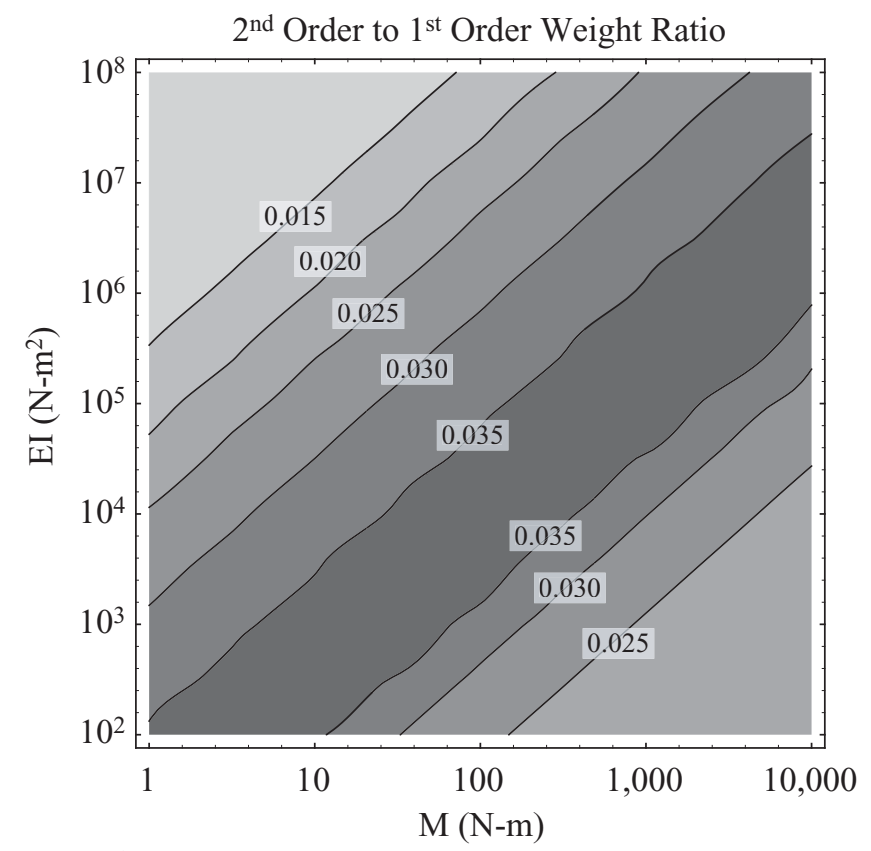

Figure 14: Ratio of $2^{\text {nd }}$ order to $1^{\text {st }}$ order truss weight per length optimization results ( $E=200$ GPa (29 Msi), $\rho$ $\left.=1660 \mathrm{~kg} / \mathrm{m}^{3}\left(0.06 \mathrm{lb} / \mathrm{in}^{3}\right)\right)$.

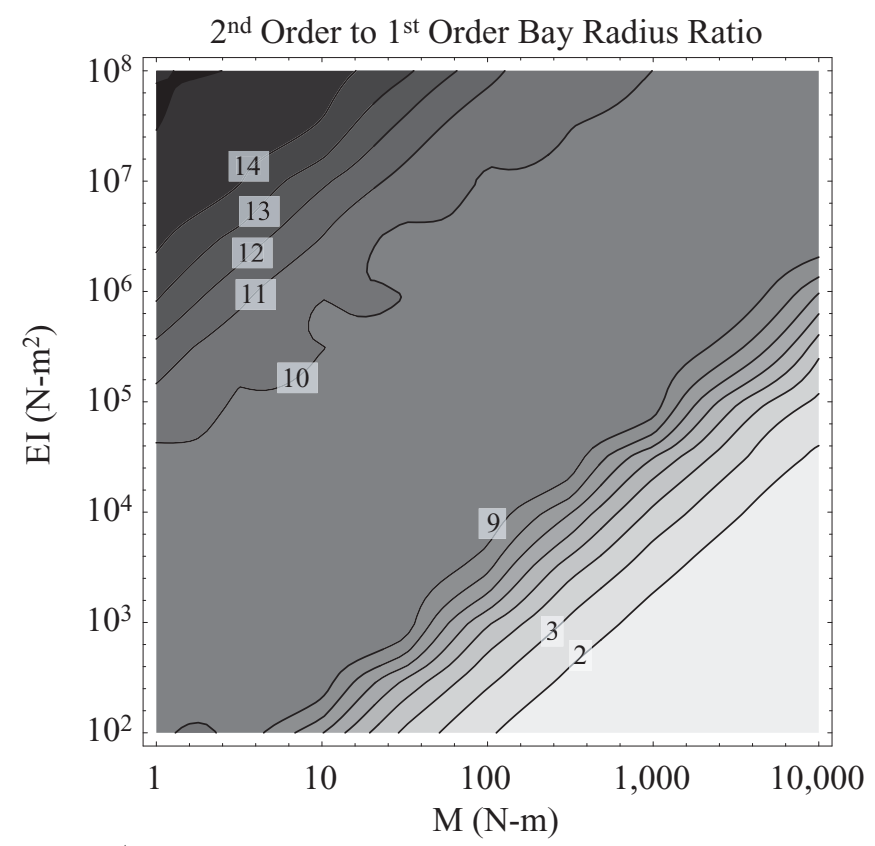

Figure 15: Ratio of $2^{\text {nd }}$ order to $1^{\text {st }}$ order truss radius optimization results ( $E=200 \mathrm{GPa}(29 \mathrm{Msi}), \rho=1660$ $\left.\mathrm{kg} / \mathrm{m}^{3}\left(0.06 \mathrm{lb} / \mathrm{in}^{3}\right)\right)$. 